\title{
GESTIÓN DEL TIEMPO Y USO DE LAS TIC EN ESTUDIANTES UNIVERSITARIOS
}

\section{MANAGEMENT OF TIME AND USE OF ICT IN UNIVERSITY STUDENTS}

\author{
Daniel Garrote Rojas ${ }^{1}$ \\ daniel.garrote@uclm.es \\ Sara Jiménez-Fernández² \\ sarajimenezfer@hotmail.com \\ Rosa María Serna Rodríguez ${ }^{1}$ \\ rosamaria.serna@uclm.es
${ }^{1}$ Departamento de Pedagogía, Facultad de Educación de Albacete, Plaza de la Universi- dad, 3, Edificio Simón Abril, CP 02071 Albacete, España
Universidad de Castilla-La Mancha
${ }^{2}$ Unidad de Salud Mental Infanto-Juvenil del Complejo Hospitalario de Jaén
Complejo Hospitalario de Jaén, Avda. de Madrid s/n C.P. 23008 Jaén, España

\begin{abstract}
Resumen: Con este trabajo de investigación pretendemos dar a conocer la incidencia de la variable sociodemográfica, edad, en el uso de internet y el teléfono móvil, la autopercepción de los estudiantes sobre ellas y la frecuencia con que su uso les causa problemas. Realizándose un estudio descriptivo sobre una muestra de estudiantes universitarios de la Facultad de Educación de la Universidad de Castilla-La Mancha. Se ha utilizado el Cuestionario de Uso Problemático de Nuevas Tecnologías (UPNT). Los resultados indican que no existen diferencias significativas para ambos grupos de edad establecidos, pero sí la gran cantidad de tiempo que les dedican tanto a diario como semanalmente a internet y el teléfono móvil. Como conclusión, destacamos la relevancia de educar en un correcto uso de las NT, para prevenir problemas derivados de su exceso de uso, así como, problemas en su entorno social. Como consecuencia del excesivo tiempo dedicado y la necesidad del uso muy frecuente por parte de la muestra, indica tendencias a la adicción en el uso de estas herramientas.
\end{abstract}

Palabras clave: Nuevas Tecnologías, Teléfono móvil, Internet, adicción.

Abstract: With this research we intend to present the incidence of the sociodemographic variable, age, in the use of the internet and the mobile phone, the students' self-perception about them and the frequency with which their use causes them problems. A descriptive study was carried out on a sample of university students from the Faculty of Education of the University of Castilla-La Mancha. The Questionnaire for the Difficult Use of New Technologies (UPNT) has been used. The results indicate that there are no significant differences for both established age groups, but the large amount of time devoted daily and weekly to the internet and mobile phone. In conclusion, we emphasize the relevance of educating in a correct use of the $N T$, to prevent problems derived from their overuse, as well as, problems in their social environment. As a consequence of the excessive time spent and the need for frequent use by the sample, it indicates tendencies to addiction in the use of these tools.

Keywords: New Tecnologies, Mobile Phone, Internet, Addiction

- 109 -

Píxel-Bit. Revista de Medios y Educación. No 53 Julio 2018. ISSN: 1133-8482. e-ISSN: 2171-7966. doi: http://dx.doi.org/10.12795/pixelbit.2018.i53.07 


\section{Introducción}

Fruto del proceso de globalización, del momento histórico en el que nos encontramos, la accesibilidad, interactividad y flexibilidad de las Tecnologías de la Información y la Comunicación (TIC) ha llevado a un mayor uso de internet, redes sociales y videojuegos en las últimas décadas, cambiando nuestra manera de trabajar, pensar y tomar decisiones.

A nivel educativo, la inclusión de las TIC en la formación que ofrecen las instituciones de enseñanza superior supone un nuevo reto, una adaptación formativa de las herramientas que tenemos y desde el Espacio Europeo de Educación Superior (EEES) se pretende dar respuesta a los estudiantes universitarios. A través del Blended-learning, se combinan los trabajos presenciales en el aula con una enseñanza a distancia, flexibilizando el proceso de aprendizaje y con el e-learning, a través de las redes informáticas se busca la enseñanza a distancia apoyada en las TIC. La tecnología no es una opción pedagógica, sino una herramienta (De Pablos y Villaciervos, 2005) que da lugar a la aparición de nuevas competencias en los Grados universitarios, dominio de las Tecnologías de la información y la Comunicación. El aprendizaje se transforma en un proceso continuo que dura toda la vida, donde las tecnologías modulan la forma de recibirlo, organizarlo y exponer lo aprendido. Considerando que el rendimiento académico de los estudiantes puede verse afectado por el uso de las TIC y tener consecuencias en su proceso de enseñanza y aprendizaje (Karpinski, Kirschner, Ozer, Mellott y Ochwo, 2013), los estudiantes deben manejarse con soltura en su uso, ya que en la universidad y en la etapa laboral, necesitarán estar familiarizados con estas herramientas para aprender y progresar (Padilla-Carmona, Suarez-Ortega y Sánchez-García, 2016). Internet y el teléfono móvil son las TIC con mayor relevancia social en su uso por parte de los estudiantes (Cruces, Guil, Sánchez y Pereira, 2016). Ofreciéndose internet como una herramienta que pone al alcance todo tipo de información, permitiendo su manipulación y ofreciendo nuevas vías de comunicación y relación. El teléfono móvil ha pasado de tener una función únicamente comunicativa por voz, a formar parte de nuestra entidad personal con la llegada de los smartphones, desde los que podemos llamar, enviar mensajes, fotos, consultar internet, escuchar música, jugar, controlar actividades deportivas, etc.

Este abanico, cada vez más amplio, de posibilidades que nos ofrecen las TIC nos llevan a nuevos estilos de relación, volviéndose un elemento indispensable. A través de las plataformas que ofrece la universidad, los docentes y discentes pueden comunicarse, planteando una nueva vía para una formación más completa de los estudiantes. Se favorece una actitud activa de estos, tanto en la recepción de la información como en su manipulación para una posterior creación (Islas y Delgadillo, 2016). Autores como González (2015) señalan que el modelo de aprendizaje del conectivismo se engrana con las necesidades de conocimiento de la sociedad porque se sirven del uso de herramientas colaborativas por parte de los estudiantes, donde los entornos virtuales de aprendizaje son el canal de estos para construir su conocimiento y poder trabajar con los compañeros. Winocur (2006) señala como el uso del teléfono móvil e internet son una herramienta de socialización on-line donde pueden ampliar sus círculos habituales de encuentro e interacción. 
La adicción que puedan crear las TIC preocupan a la sociedad, especialmente internet y el uso del teléfono móvil. La adicción se define como la dependencia a sustancias o actividades nocivas para la salud, donde hay una búsqueda y consumo compulsivo del objeto de adicción, independientemente de sus consecuencias nocivas. Por tanto, la adicción a las nuevas tecnologías consiste en la búsqueda y uso compulsivo de los recursos tecnológicos, con repercusión en la calidad de vida de los sujetos. los usuarios llegan a tener un síndrome de abstinencia cuando no pueden hacer uso de ella, que cursa con malestar emocional (Echeburúa y Corral, 2010). En este sentido, en el Manual Diagnóstico y Estadístico de los Trastornos Mentales (DSM-V), en el apartado de los Trastornos relacionados con sustancias y trastornos adictivos se hace referencia únicamente al juego patológico. Por ejemplo, el uso excesivo de los videojuegos en jóvenes, puede llevar al desarrollo de hábitos difíciles de modificar que generan efectos negativos a nivel físico, cognitivo y social (Chacón, Zurita, Martínez, Castro, Espejo y Pinel, 2017). Coincidiendo con Kennedy et al. (2010), debe reivindicarse en la investigación un análisis de los diferentes elementos implicados en el uso de las TIC que realizan los estudiantes más que buscar las diferencias generacionales. Se están llevando a cabo diferentes investigaciones para conocer si estamos ante una nueva modalidad de adicción (Muñoz-Rivas y Agustín, 2005; Carbonell, Chamarro, Griffiths, Oberst, Cladellas y Talarn, 2012).

El uso de las TIC como herramienta de aprendizaje, ofrece una perspectiva motivadora que influye en el desarrollo del aprendizaje de los estudiantes (García-Cué, Santizo y Alonso, 2009). Esta investigación pretende conocer el tiempo que dedican a las TIC y si estas actividades puede generarles problemas importantes como la pérdida de tiempo para realizar otras actividades, un gasto económico significativo o incluso ser motivo de discusiones con familia o amigos.

\section{Objetivos}

A través de este trabajo de investigación pretendemos alcanzar cinco objetivos, conocer el tiempo que dedican los estudiantes universitarios al uso del teléfono móvil cada día y semanalmente, conocer el tiempo que dedican los estudiantes universitarios a internet a diario y semanalmente, identificar las características que presenta la muestra en su uso y describir los diferentes patrones de consumo entre los mayores y menores de 22 años.

\section{Método}

Se ha llevado a cabo un trabajo de investigación cuantitativo mediante la aplicación de un cuestionario. Se realiza un análisis de carácter descriptivo mediante el uso de medidas de tendencia central y medidas de dispersión sobre el valor obtenido en cada ítem por los estudiantes según dos rangos de edad, los menores o igual de 21 años (76,9\% de la muestra) y los mayores de 22 años (23,1\% de la muestra), utilizando como criterio la edad media de la muestra (20.94 \pm 3.38).

El análisis estadístico de los datos recogidos en la investigación se ha llevado a cabo a través del programa Statisfical Package for the Social Science (SPSS) versión 22.0. 


\subsection{Diseño y participantes}

Para el desarrollo de esta investigación se ha llevado a cabo un diseño descriptivo y de corte transversal. Participaron 108 estudiantes universitarios $(n=108)$ del Grado de Maestro en Educación Infantil y Primaria. La media de edad de los alumnos fue de 21 años. Mayoritariamente del género femenino (94.4\%). Se utilizó el cuestionario de Uso Problemático de Nuevas Tecnologías (UPNT) para la recogida de la información.

El criterio de selección utilizado fue ser estudiante matriculado del curso académico 2015/2016 del Grado de Maestro en Educación, como criterio de exclusión, todos aquellos estudiantes que no estuvieron presentes en la recogida de los datos. La participación fue voluntaria, tras consentimiento informado verbal sobre la investigación que se estaba llevando a cabo y tras garantizando el anonimato de los participantes. Se informó que el uso de los datos tiene exclusivamente fines investigativos.

\subsection{Variables e instrumentos}

Se utilizó el Cuestionario de Uso Problemático de Nuevas Tecnologías (UPNT), Labrador, Villadandos, Crespo y Becoña (2013). Está formado por 41 ítems, los dos primeros hacen referencia a la frecuencia y problemas del uso de las nuevas tecnologías, posteriormente, hay cuatro subescalas relacionados con el uso de Internet, los Videojuegos, Teléfono móvil y el uso de la Televisión. Los cuatro primeros factores se refieren al uso de cada una de las Nuevas Tecnologías (NT) evaluada (Teléfono

\section{Con}

\begin{tabular}{|c|c|c|c|c|c|c|c|c|c|c|c|c|c|}
\hline \multirow{3}{*}{ NT } & \multirow{3}{*}{ Edad } & \multicolumn{2}{|c|}{ Nunca } & \multicolumn{2}{|c|}{ A veces } & \multicolumn{2}{|c|}{ frecuencia } & \multicolumn{2}{|c|}{ Siempre } & \multirow{2}{*}{\multicolumn{4}{|c|}{ Total }} \\
\hline & & & 1) & & (2) & & (3) & & 4) & & & & \\
\hline & & $\mathbf{N}$ & $\%$ & $\mathbf{N}$ & $\%$ & $\mathbf{N}$ & $\%$ & $\mathbf{N}$ & $\%$ & $\%$ & $\mathbf{N}$ & Media & $\mathbf{S}$ \\
\hline & $=21$ & 37 & 44,6 & 23 & 27,7 & 18 & 21,7 & 3 & 3,6 & 97,6 & 81 & 1,84 & ,901 \\
\hline & $>22$ & 15 & 60,0 & 4 & 16,0 & 3 & 12,0 & 2 & 12,0 & 100 & 25 & 1,76 & 1,091 \\
\hline & Total & 52 & 49,1 & 27 & 25,5 & 21 & 19,8 & 6 & 5,7 & 98,2 & 106 & 1,82 & .944 \\
\hline \multirow{3}{*}{ 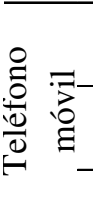 } & $=21$ & 18 & 21,7 & 31 & 37,3 & 24 & 28,9 & 8 & 9,6 & 97,6 & 81 & 2,27 & ,922 \\
\hline & $>22$ & 4 & 16,0 & 11 & 44,0 & 7 & 28,0 & 3 & 12,0 & 100 & 25 & 2,36 & ,907 \\
\hline & Tota & 22 & 20,8 & 42 & 39,6 & 31 & 25,2 & & 4 & & & & \\
\hline
\end{tabular}

Tabla 1. Estadísticos descriptivos y frecuencias de los ítems 6-10 sobre la frecuencia con que causan problemas por dedicar exceso de tiempo. 


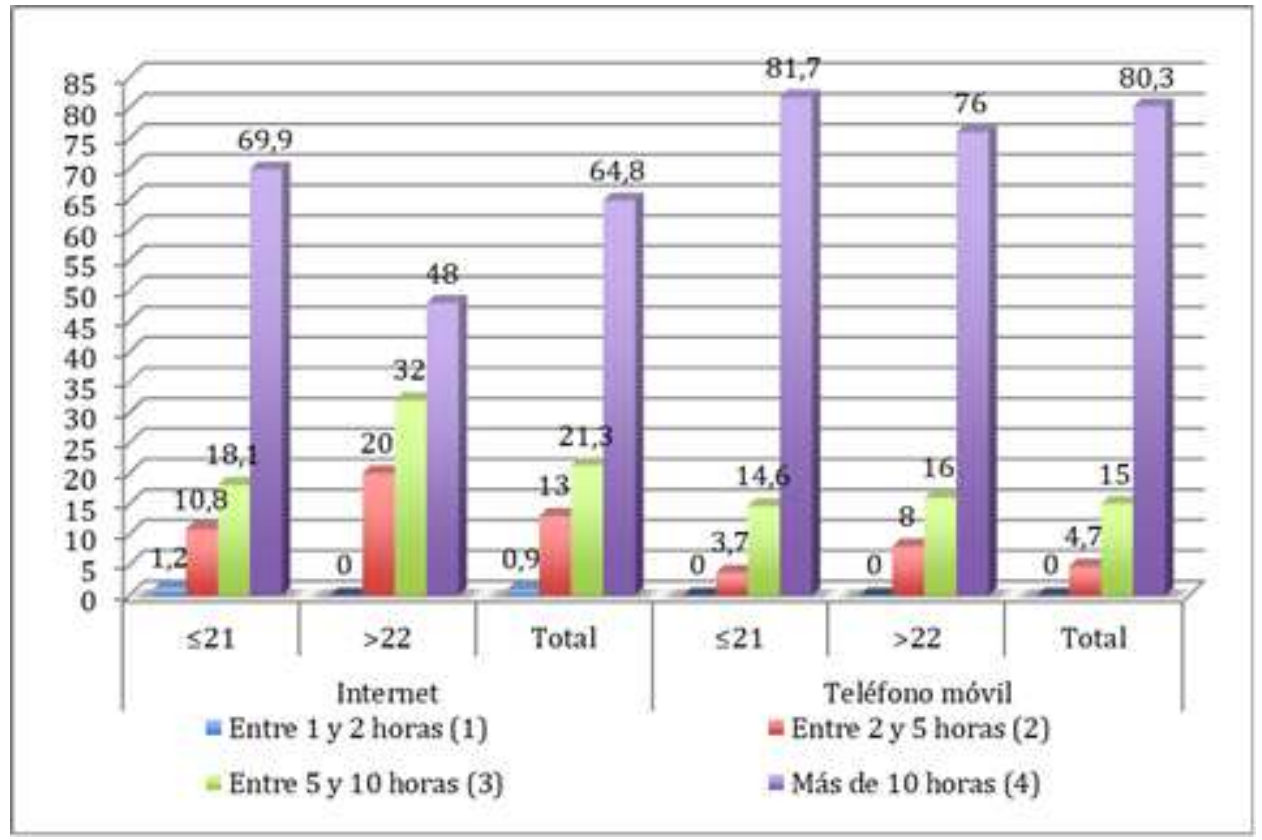

Gráfico 1. Frecuencias sobre las horas dedicadas a la semana.

móvil, Videojuegos, Televisión e Internet), los demás factores se centran en identificar las conductas características de utilización problemáticas de las mismas, mentir sobre su uso, relajarse con el mismo e intentar frustradamente dejar de utilizarlas. Los índices de fiabilidad del cuestionario muestran un alfa de Cronbach de .876 en la escala total y las cuatro subescalas oscilan entre .713 y .876. Igualmente, presenta una adecuada validez clínica.

El tipo de respuesta varía en función de los ítems, mostrando diferentes alternativas de respuesta, en algunos casos entre: «nunca», «alguna vez al mes», «alguna vez a la semana» $\mathrm{y}$ «todos los días». En otros casos: «nunca», «a veces», «con frecuencia»y «siempre». Respecto a la cantidad de horas dedicadas al día varía de: «1 a 3 horas», «3 a 5 horas» y «Más de 5 horas». En relación a las horas dedicadas a la semana la escala es entre: «1 y $2 », \ll 2$ y $5 », \ll 5$ y $10 »$ y «Más de $10 »$.

\section{Resultados}

Los participantes que formaron parte de la investigación fueron 108 estudiantes, $94.4 \%$ mujeres, con una edad media de $20.94 \pm 3.38$. El 76.9\% de los estudiantes está entre los 18 y 21 años. La media de edad se ha utilizado como criterio para separar la muestra en dos grupos: el primer grupo, aquellos con una de edad menor o igual a 21 años (desde los 18 a los 21 años) y un segundo grupo con una edad mayor de 22 años (desde los 22 a los 41). Respecto a la frecuencia de utilización de las Nuevas Tecnologías (NT), internet es utilizado por el $99.1 \%$ de la muestra todos los días y el $100 \%$ alguna vez a la semana, siendo la puntuación media de $3.99 \pm 0.09$. El teléfono 


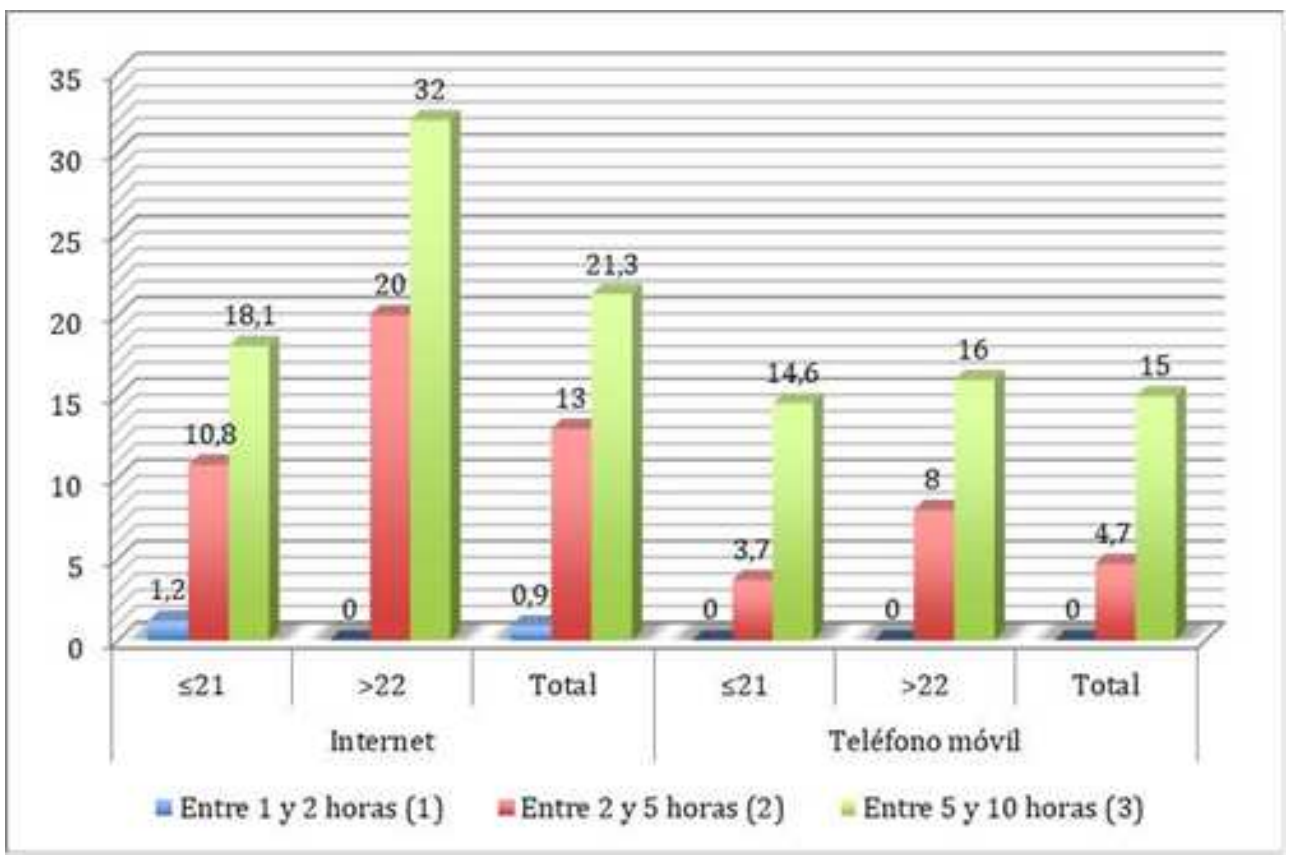

Gráfico 2. Frecuencias sobre las horas dedicadas al día.

móvil lo utiliza el $100 \%$ de la muestra todos los días, siendo la puntuación media de 4.00.

En la tabla 1 aparece la frecuencia, la media y la desviación típica con que el uso de estas NT (internet y teléfono móvil) causan problemas por una dedicación excesiva. La puntuación media obtenida en los sujetos menores de 21 años oscila entre 1.00 y 2.27 , en los sujetos mayores de 22 años, 1.08 y 2.36 y del total de la muestra, 1.02 y 2.29 . El uso del teléfono móvil es la actividad que mayor problema causan a los estudiantes.

Según la frecuencia con que les causan problemas el uso de las NT, los datos indican que, en el uso del teléfono móvil, el 39.6\% de la muestra les producen problemas con frecuencia o siempre. En el uso de internet, el $50.9 \%$ de la muestra han tenido alguna vez problemas por su uso. En función de la edad, la escala de las actividades decreciente en función de qué le causa problemas para el grupo d»21 años son teléfono móvil e internet, así como para los $>22$ años.

Respecto a las características de uso del teléfono móvil, el $81,5 \%$ de los estudiantes dicen sentirse mal al no poder utilizarlo, el $56,6 \%$ piensa horas antes de usar el móvil en él y el 16,7\% llega a mentir a la familia o amigos sobre las horas que dedica al móvil. El 59,3\% alguna vez ha intentado dejar el uso del móvil y no lo has conseguido. Al 88\% de los estudiantes le relaja utilizar el teléfono móvil, y el $55,1 \%$ se siente nervioso si pasa mucho tiempo desde la última vez que usó el teléfono móvil. En relación a las características de uso de internet, el 90,7\% considera que dedica más tiempo del que cree necesario a internet, el 69,4\% se encuentra mal cuando por algún motivo no puede utilizar internet, el 38\% piensa desde horas antes de conectarte a 


\section{Con}

\section{Nunca A veces frecuencia Siempre}

\begin{tabular}{|c|c|c|c|c|c|c|c|c|c|c|c|c|c|}
\hline \multirow[t]{2}{*}{ Ítem } & \multirow[t]{2}{*}{ Edad } & \multicolumn{2}{|c|}{ (1) } & \multicolumn{2}{|r|}{ (2) } & \multicolumn{2}{|c|}{ (3) } & \multicolumn{2}{|c|}{ (4) } & \multicolumn{4}{|c|}{ Total } \\
\hline & & $\mathbf{N}$ & $\%$ & $\mathbf{N}$ & $\%$ & $\mathbf{N}$ & $\%$ & $\mathbf{N}$ & $\%$ & $\%$ & $\mathbf{N}$ & Media & $\mathbf{s}$ \\
\hline \multirow{3}{*}{27} & $=21$ & 15 & 18,1 & 45 & 54,2 & 16 & 19,3 & 7 & 8,4 & 100 & 83 & 2,18 & ,829 \\
\hline & $>22$ & 5 & 20,0 & 14 & 56,0 & 4 & 16,0 & 2 & 8,0 & 100 & 25 & 2,12 & ,833 \\
\hline & Total & 20 & 18,5 & 59 & 54,6 & 20 & 18,5 & 9 & 8,3 & 100 & 108 & 2,17 & 826 \\
\hline \multirow{3}{*}{28} & $=21$ & 35 & 42,2 & 35 & 42,2 & 11 & 13,3 & 2 & 2,4 & 100 & 83 & 1,76 & ,774 \\
\hline & $>22$ & 12 & 48,0 & 9 & 36,0 & 3 & 12,0 & 1 & 4,0 & 100 & 25 & 1,72 & 843 \\
\hline & Total & 47 & 43,5 & 44 & 40,7 & 14 & 13,0 & 3 & 2,8 & 100 & 108 & 1,75 & ,787 \\
\hline \multirow{3}{*}{29} & $=21$ & 70 & 84,3 & 10 & 12,1 & 2 & 2,4 & 1 & 1,2 & 100 & 83 & 1,20 & ,536 \\
\hline & $>22$ & 20 & 80,0 & 3 & 12,0 & 1 & 4,0 & 1 & 4,0 & 100 & 25 & 1,32 & ,748 \\
\hline & Total & 90 & 83,3 & 13 & 12,0 & 3 & 2,8 & 2 & 1,9 & 100 & 108 & 1,23 & ,590 \\
\hline \multirow{3}{*}{30} & $=21$ & 32 & 38,6 & 35 & 42,2 & 14 & 16,9 & 2 & 2,4 & 100 & 83 & 1,83 & ,794 \\
\hline & $>22$ & 12 & 48,0 & 8 & 32,0 & 4 & 16,0 & 1 & 4,0 & 100 & 25 & 1,76 & 879 \\
\hline & Total & 44 & 40,7 & 43 & 39,8 & 18 & 16,7 & 3 & 2,8 & 100 & 108 & 1,81 & 811 \\
\hline \multirow{3}{*}{31} & $=21$ & 10 & 12,1 & 42 & 50,6 & 19 & 22,9 & 12 & 14,5 & 100 & 83 & 2,40 &, 883 \\
\hline & $>22$ & 3 & 12,0 & 17 & 68,0 & 4 & 16,0 & 1 & 4,0 & 100 & 25 & 2,12 & ,666 \\
\hline & Total & 13 & 12,0 & 59 & 54,6 & 23 & 21,3 & 13 & 12,0 & 100 & 108 & 2,33 & ,843 \\
\hline \multirow{3}{*}{32} & $=21$ & 40 & 48,8 & 26 & 31,7 & 9 & 11,0 & 7 & 8,5 & 100 & 82 & 1,79 & ,952 \\
\hline & $>22$ & 8 & 32,0 & 10 & 40,0 & 6 & 24,0 & 1 & 4,0 & 100 & 25 & 2,00 & ,866 \\
\hline & Total & 48 & 44,9 & 36 & 33,6 & 15 & 14,0 & 8 & 7,5 & 100 & 108 & 1,84 & ,933 \\
\hline
\end{tabular}

Tabla 2. Estadísticos descriptivos y frecuencias de los ítems 27-32 sobre el uso del teléfono móvil

Píxel-Bit. Revista de Medios y Educación. No 53 Julio 2018. ISSN: 1133-8482. e-ISSN: 2171-7966. doi: http://dx.doi.org/10.12795/pixelbit.2018.i53.07 
internet en ello. El 12\% miente a la familia o amigos sobre las horas que dedicas a utilizar internet y el 32,4\% alguna vez ha intentado desconectarte de internet y no lo ha conseguido. El 81,5\% utiliza internet como modo de relajarse y el. $37 \%$ se sientes nervioso si pasa mucho tiempo desde la última vez que estuviste conectado a internet.

En relación a la frecuencia de uso de las NT, en el gráfico 1 podemos observar las horas que dedican los estudiantes a la semana a las NT. La mayor frecuencia de uso se da en el teléfono móvil, donde el 80,3\% dedica más de 10 horas a la semana, seguido del uso de internet con un $64,8 \%$ de los estudiantes. Siendo las medías obtenidas para los sujetos menores de 21 años y mayores de 22 años similares.

Analizando de manera descendente el tiempo que dedican diariamente a las NT, en el gráfico 2 podemos observar como el 85,1\% de los estudiantes dedican más de 3 horas al teléfono móvil, de los que el $47,7 \%$ supera las 6 horas diarias. Los menores de 22 años dedican más tiempo $(5,12$ horas $)$ que los mayores de 22 años (4,12 horas). El acceso a internet es utilizado más de 3 horas por el $65,4 \%$, de los que el $25,2 \%$ indica que el tiempo que pasan es superior a 6 horas. Encontrando diferencias en función de la edad, en el uso de internet, los menores de 21 años obtienen una puntuación media de 2,11 y los mayores de 22 años una puntuación media de 1,76. En el uso del teléfono móvil, los menores de 21 años obtienen una puntuación media de 2,50 y los mayores de 22 años una puntuación media de 2,24.

Centrándonos en el uso del teléfono móvil e internet. El primero está formado por seis ítems. En la tabla 2 observamos como en el ítem 27: «Te encuentras mal cuando por algún motivo no puedes usar el teléfono móvil?», el $81,5 \%$ ha respondido afirmativamente, de los que el 54,6\% le sucede a veces, el 18,5\% con frecuencia y el 8,3\% siempre. En el ítem 28: «¿Estás pensando desde horas antes de usar el móvil?», el 43,5\% del total de la muestra ha respondido negativamente, del $56,6 \%$ que ha respondido afirmativamente, el $40,7 \%$ ha respondido «a veces», el $13 \%$ que «con frecuencia» y el 2,8\% «siempre». En el ítem 29: «¿Mientes a tu familia o amigos sobre las horas que dedicas al móvil?», el 16,7\% dice que sí, de los que el $12 \%$ lo hace «a veces», el 2,8\% «con frecuencia» y el 1,9\% «siempre». Para el ítem 30: «¿Alguna vez has intentado dejar el móvil y no lo has conseguido?», el $59,3 \%$ responde afirmativamente, el 39,8\% «a veces», el 16,7\% «con frecuencia» y el 2,8\% «siempre». En el ítem 31: «¿Te relaja usar el móvil?», el 88\% responde que sí, de los que el 54,6\% responde que «a veces», el 21,3\% «con frecuencia y el $12,2 \%$ «siempre». Al diferenciar por edad, los menores de 21 años, el 14,5\% ha respondido «siempre»y de los mayores de 22 años, el $4 \%$ ha dado la misma respuesta. Por último, en el ítem 32: « ¿Te sientes nervioso si pasa mucho tiempo desde la última vez que usaste el móvil?», el 55,1\% responde afirmativamente, de los que $33,6 \%$ indica que «a veces», el 14,0\% «con frecuencia»y el $7,5 \%$ «siempre».

En relación al uso de internet, como se indica en la tabla 3, está formado por siete ítems. El ítem 6: «¿Dedicas más tiempo del que crees necesario a internet?», el 9,3\% indica que no. De la muestra que responde afirmativamente, el 42,6\% indica que «a veces», 30,6\% «con frecuencia»y el 17,6\% «siempre».

El ítem 7: « $i$ Te encuentras mal cuando por algún motivo no puedes utilizar internet queriendo hacerlo?», el 30,6\% indica que no, 


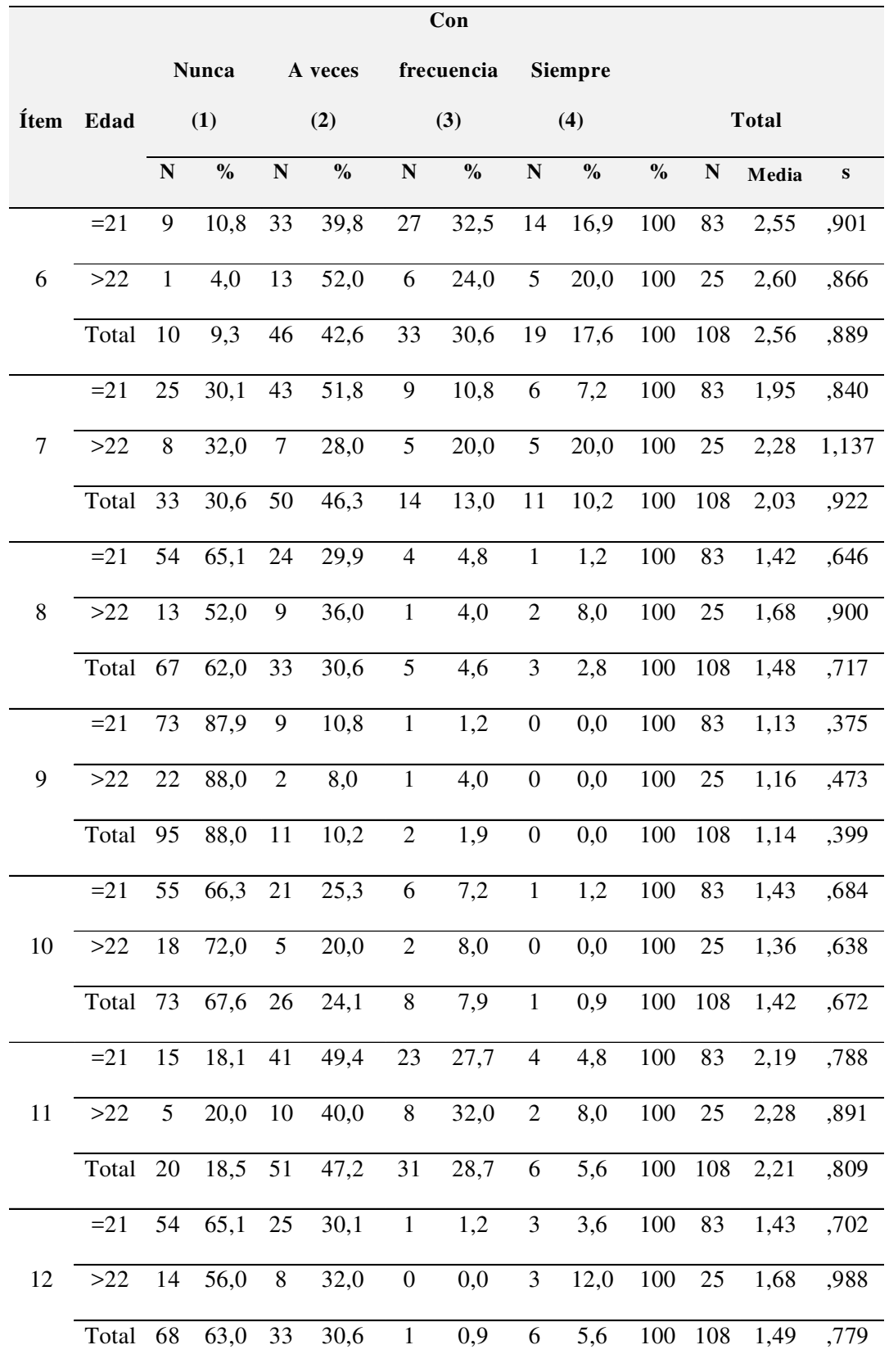

Tabla 3. Estadísticos descriptivos y frecuencias de los ítems 6-12 sobre el uso de Internet 
el 46,3\% «a veces», el 13\% «con frecuencia» y el $20 \%$ «siempre».

El ítem 8: «¿Estás pensando desde horas antes de conectarte a internet en ello?», el $38 \%$ ha respondido que sí, pero en diferente grado, el 30,6\% «a veces», el 4,6\% «con frecuencia»y el 2,8\% «siempre».

En el ítem 9: «¿Mientes a tu familia o amigos sobre las horas que dedicas a utilizar internet?», un $12 \%$ de la muestra reconoce que sí, el 10,2\% lo hace «a veces» y el 1,8\% «con frecuencia».

Para el ítem 10: «¿Alguna vez has intentado desconectarte de internet y no lo has conseguido?», el 67,7\% responde que no, el $24,1 \%$ indica que «a veces», el 7,9\% «con frecuencia»y el 0,9\% «siempre».

En el ítem 11: « ¿Te relaja navegar por internet?», el $81,5 \%$ respondió afirmativamente, el 47,2\% indica «a veces», el $28,7 \%$ «con frecuencia» y el 5,6\% «siempre».

Por último, en el ítem 12: «¿Te sientes nervioso si pasa mucho tiempo desde la última vez que estuviste conectado a internet?», el 63\% indica que no y del 37\% que responde que sí, el 30,6\% lo hace «a veces», el 0,9 «con frecuencia» y el 5,6\% «siempre».

\section{Conclusiones y discusión}

Considerando los objetivos planteados en esta investigación y el análisis estadístico llevado a cabo sobre el uso de las nuevas tecnologías desde un punto de vista no formal, es decir, cuando no persigue necesariamente una finalidad académica. La frecuencia de uso diario en Internet y el teléfono móvil es del 99,1\%. En el caso de Internet, se ha producido un aumento sustancial en comparación con otra investigación (Graner, Beranuy, SánchezCarbonell, Chamarro y Castellana, 2007), donde el 58,4\% de la muestra indicó que se conectaba diariamente. Este aumento puede deberse al aumento en la accesibilidad que ha habido a través de diferentes dispositivos como los smartphones. La distribución de la frecuencia de uso diaria de las tecnologías analizadas en un uso mayor de cinco horas de forma decreciente son: Teléfono móvil $(47,7 \%)$ e Internet $(25,2 \%)$. Y entre tres y cinco horas: Internet $(40,2 \%)$ y Teléfono móvil $(37,4 \%)$. Por tanto, la frecuencia de uso diaria superior a tres horas es: Teléfono móvil $(85,1 \%)$ e Internet $(65,4 \%)$. En la investigación llevada a cabo por Ortiz (2013), el 42,1\% de los estudiantes universitarios se conectan a internet entre 1 y 2 horas al día, mientras que en nuestro caso, el 40,2\% se conecta entre 3 y 5 horas.

En relación a la frecuencia de uso semanal de las tecnologías con una dedicación mayor de 10 horas son: Teléfono móvil $(80,3 \%)$ e Internet (64,8\%). En la investigación llevada a cabo por Muñoz-Rivas, Navarro-Perales y De Pablo (2003) el 9,9\% de los participantes usaban internet más de 10 horas a la semana, mientras que en nuestra investigación son el 64,8\%.

El 50,9\% ha presentado algún problema por dedicación excesiva de tiempo, en comparación con otras investigaciones, el 44,5\% (Cruces, Guil, Sánchez y Pereira, 2016), el 19,5\% (Ortiz, 2013) y el 19,9\% (Carbonell, Chamarro, Griffiths, Oberst, Cladellas y Talarn, 2012). El 5,7\% de la muestra presenta problemas siempre y el $19,8 \%$ con frecuencia. Coincidiendo con el 5,7\% en el estudio de Cruces, Guil, Sánchez y Pereira, (2016). Dicho problema se presenta en el 2,2\% para Carbonell, Chamarro, Griffiths, Oberst, Cladellas y Talarn (2012), el 3,2\% para Kuss, 
Griffiths y Binder (2013) y el 9,9 para MuñozRivas, Fernández y Gámez-Guadix (2010).

Las características de uso del teléfono móvil indican un elevado número de sujetos que se encuentran mal cuando no pueden utilizarlo, así como una posible ansiedad anticipatoria, incluso en ocasiones mienten al indicar la cantidad de tiempo que les dedican. A la mitad de la muestra les cuesta apagar o desconectarse de su uso. Como hemos indicado anteriormente, el análisis de este tipo de comportamiento nos reporta información relevante que podría ser igualmente válida y comparable para sujetos adictos a alguna sustancia. Según Pedrero, Rodríguez y Ruíz (2012), el uso del teléfono móvil puede llegar a transformarse en un comportamiento disfuncional. Cuando se produce el cambio en la conducta de estar su uso bajo el control consciente y se vuelve un comportamiento automatizado, puede ser considerado como un criterio de adicción (Everitt y Robbins, 2005). Olivencia-Carrión, Pérez-Marfil, Ramos-Revelles y LópezTorrecillas (2016) indican que hay una alta relevancia de los factores psicológicos en la adicción al teléfono móvil al pensar los usuarios que su uso les supone una mejora en su vida, como la vida social, sin embargo, otros pueden verlo como una pérdida de tiempo y dinero. Una amplia mayoría lo utiliza como forma de relajamiento. Según Chóliz (2012), el uso del teléfono móvil puede verse como una estrategia de afrontamiento del estado emocional negativo como el aburrimiento. A la mitad de los usuarios les crea incertidumbre si pasa mucho tiempo sin verlo. Llegándose a acuñar el término «era del pulgar» para explicar un comportamiento intensivo, permanente, invasivo y omnipresente, donde los usuarios pasan más tiempo mirando su teléfono móvil que cualquier otro objeto (Bell, 2005; Park, 2005).

En relación a las características de uso de internet, El 90,7\% considera que dedica más tiempo del que cree necesario a internet. El $69,4 \%$ se encuentras mal cuando por algún motivo no pueden utilizar internet queriendo hacerlo, puediendo deberse como describen Gil, del Valle, Oberst y Chamorro (2015) al fear of missing out (miedo a perderse algo) como causa plausible. Descrito como una sensación de malestar al pensar que otras personas están realizando actividades agradables en las que uno no forma parte de ella. Esta accesibilidad permanente puede crear en los usuarios una constante necesidad de búsqueda de información. En ocasiones, pudiendo llevarles a una revisión permanente de sus aplicaciones (como por ejemplo, redes sociales) para estar actualizados con los intereses, gustos y planificaciones de su círculo de amistades.

Existen algunas diferencias entre ambos grupos de edad establecidos. En la frecuencia de uso del teléfono móvil, los menores de 22 años dedican al día una hora más al teléfono móvil que los mayores de 22 años. En el acceso a internet, también se produce esta diferencia a favor de los menores de 22 años en casi una hora diaria frente a los mayores de 22 años. Ante la relevancia de las consecuencias de un uso excesivo del teléfono móvil y de internet y de la posible aparición de una adicción en edades tempranas de la etapa adulta, sería conveniente ampliar el estudio sobre el uso de las NT y analizar cómo educar a la población en un uso responsable donde se de paso a una correcta gestión de las herramientas que hay a nuestro alcance. Considerando que la oferta, cada vez mayor, de diferentes aplicaciones para incorporar en 
cada actividad que llevemos a cabo de trabajo u ocio, así como el uso de las redes sociales puede hacer difícil mantener un equilibrio entre su uso y nuestra independencia.

\section{REFERENCIAS}

American Psychiatric Association. (2013). Guía de consulta de los criterios diagnósticos del DSM-V. Madrid: Editorial médica panamericana.

Bell, G. (2005). The age of the thumb: a cultural reading of mobile technologies from Asia. En P. Glotz, S. Bertschi y C. Locke (eds.), Thumb culture: the meaning of mobile phones for society (pp. 67-87). Bielefeld: Deutsche Bibliothek.

Carbonell, X., Chamarro, A., Griffiths, M., Oberst, U., Cladellas, R. y Talarn, A. (2012). Problematic Internet and cell pone use in Spanish teenagers and Young students. Anales de Psicología, 28(3), 789-796.

Chacón, R., Zurita, F., Martínez, A., Castro, M., Espejo, T. y Pinel, C. (2017). Relación entre factores académicos y consumo de videojuegos en universitarios. Un modelo de regresión. Pixel-Bit. Revista de Medios y Educación, 50, 109-121.

Doi: http://dx.doi.org/10.12795/ pixelbit.2017.i50.07

Chóliz, M. (2012). Mobile-phone addiction in adolescence: The Test of Mobile Phone Dependence (TMD). Progress in Health Sciences, 2, 33-44.

Cruces, J., Guil, R., Sánchez, N. y Pereira, J. A. (2016). Consumo de nuevas tecnologías y factores de personalidad en estudiantes universitarios. Revista de Comunicación y Ciudadanía Digital-COMMONS, 5 (2), 203228.

De Pablos, J. y Villaciervos, P. (2005). El Espacio Europeo de Educación Superior y las
Tecnologías de la Información y la Comunicación. Percepciones y demandas del profesorado. Revista de Educación, 227, 99124.

Echeburúa, E. Y Corral, P. (2010). Adicción a las nuevas tecnologías y a las redes sociales en jóvenes. Adicciones, 22(2), 91-96.

Everitt, B. J. y Robbins, T. W. (2005). Neural systems of reinforcement for drug addiction: from actions to habits to compulsion. Nature Neuroscience, 8, 1481-1489.

Doi:10.1038/nn1579

García-Cué, J. L., Santizo, J. A. y Alonso, C. M., (2009). Uso de las TIC de acuerdo a los estilos de aprendizaje de docentes y discentes. Revista Iberoamericana de Educación, 48(2), 1-14.

Gil, F., del Valle, G., Oberst, U. y Chamorro, A. (2015). Nuevas tecnologías- ¿Nuevas patologías? El Smartphone y el fear of missing out. Aloma, 33(2), 77-83.

González, M. (2015). Enriquecimiento tecnológico y psicopedagógico del concepto de comunidades de práctica en la educación a distancia. Revista de Educación a Distancia, 47(7), 1-22

Doi: $10.6018 / \mathrm{red} / 47 / 7$

Graner, P., Beranuy, M., Sánchez-Carbonell, X., Chamarro, A. y Castellana, M. (2007). ¿Qué uso hacen los jóvenes y adolescentes de internet y el móvil? Comunicación E Xuventude: Actas do Foro International, 7190.

Islas, C. y Delgadillo, O. (2016). La inclusión de TIC por estudiantes universitarios: una mirada desde el conectivismo. Apertura, 8(2), 116-129.

Doi:http://dx.doi.org/10.18381/ Ap.v8n2.845

Karpinski, A. C., Kirschner, P. A., Ozer, I., Mellott, J. A. y Ochwo, P. (2013). An exploration of social networking site use, 
multitasking and academic performance among United States and European University students. Computers in Human Behavior, 29(3), 1182-1192.

Doi: 10.1016/j.chb.2012.10.011.

Kennedy, G., Judd, T., Dalgarnot, B., y Waycott, J. (2010). Beyond natives and immigrants: exploring types of net generation students, Journal of Computer Assisted Learning, 26, 332-343.

Doi: 10.1111/j.1365-2729.2010.00371.x

Kuss, D.J., Griffiths M.D. y Binder, J.F. (2013). Internet addiction in students: Prevalence and risk factors. Computers in Human Behavior, 29(3), 959-966.

Doi:http://dx.doi.org/10.1016/ j.chb.2012.12.024

Labrador, F.J., Villadandos, S.M., Crespo, M. y Becoña, E. (2013). Desarrollo y validación del cuestionario de uso problemático de nuevas tecnologías (UPNT(. Anales de psicología, 29(3), 836-847.

Doi: 10.6018/analesps.29.3.159291

Muñoz-Rivas, M. y Agustín, S. (2005). LA adicción al teléfono móvil. Psicología Conductual, 13, 481-493.

Muñoz-Rivas, M.J., Navarro-Perales, M.E. y De Pablo, N.O. (2003). Patrones de uso de internet en Población Universitaria Española. Adicciones, 15(2), 137-144.

Muñoz-Rivas, M.J., Fernández, L. y GámezGuadix, M. (2010). Analysis of the indicators of pathological Internet use in Spanish university students.The Spanish Journal of Psychology, 13(2), 697-707.

Olivencia-Carrión, M.A., Pérez-Marfil, M.N., Ramos-Revelles, M.B. y LópezTorrecillas F. (2016) Personalidad y su relación con el uso versus abuso del teléfono móvil. Acción Psicológica, 13(1), 109-118.

Doi: http://dx.doi.org/10.5944/ap.13.1.17427
Ortiz Gómez, M.M. (2013). Caracterización y evaluación del uso desadaptativo de internet en estudiantes universitarios. Granada: Universidad de Granada.

Padilla-Carmona, M.T., Suarez-Ortega, M. y Sánchez-García, M.F. (2016). Inclusión digital de los estudiantes adultos que acceden a la universidad: análisis de sus actitudes y competencias digitales. Revista Complutense de Educación, 27(3), 1229-1246.

Doi:http://dx.doi.org/10.5209/ rev_RCED.2016.v27.n3.47669

Park, W. K. (2005). Mobile phone addiction. En R. Ling y P. E. Pedersen (Eds.), Mobile communications: renegotiation of the social sphere (pp. 253-270). Londres: SpringerVerlag.

Pedrero, E., Rodríguez, M.T. y Ruíz, J.M. (2012). Adicción o abuso del teléfono móvil. Revisión de la literatura. Adicciones, 24(2), 139-152.

Winocur, R. (2006). Internet en la vida cotidiana de los jóvenes. Revista mexicana de sociología, 68(3), 551-580.

Fecha de recepción: 09-03-2017

Fecha de evaluación: 28-03-2017

Fecha de aceptación: 03-04-2017 